\title{
Spin-Excitation-Instability-Induced Quantum Phase Transitions in Double-Layer Quantum Hall Systems
}

\author{
Lian Zheng, R.J. Radtke, and S. Das Sarma \\ Department of Physics, University of Maryland, College Park, Maryland 20742-4111
}

(June 25, 2021)

\begin{abstract}
We study intersubband spin density collective modes in double-layer quantum Hall systems at $\nu=2$ within the time-dependent Hartree-Fock approximation. We find that these intersubband spin density excitations may soften under experimentally accessible conditions, signaling a phase transition to a new quantum Hall state with interlayer inplane antiferromagnetic spin correlations. We show that this novel canted antiferromagnetic phase is energetically stable and that the phase transition is continuous.
\end{abstract}

73.40.Hm 73.20.Mf 73.20.Dx

Typeset using REVTEX 
Electron systems in confined geometries exhibit a richer variety of physical properties than their higher-dimensional counterparts due to enhanced interaction effects in reduced dimensions. Interaction in a low-dimensional system does not merely result in stronger renormalization of physical quantities, but can in many cases drive the system into completely new phases with peculiar properties. For a two-dimensional electron gas in a perpendicular magnetic field, the interaction effects are especially important because of Landau level quantization. When electrons are entirely restricted to the lowest Landau level by a large magnetic field, electron-electron interaction completely dominates the properties of the system as the electron kinetic energy is quenched to an unimportant constant. One of the most interesting phenomena in this strongly-correlated system is the quantum Hall effect (QHE), which has attracted a great deal of experimental and theoretical interest during the last fifteen years [1]. Recent advances in materials growth techniques have made it possible to fabricate high-quality double-layer two-dimensional electron systems with the electrons confined to two parallel planes separated by a distance comparable to that between electrons within a plane. With the introduction of this layer degree of freedom, many qualitatively new effects due entirely to interlayer correlations appear 2 . In this letter, we present a theoretical study of the intersubband spin-density-wave (SDW) excitations and the associated phase transitions in double-layer electron systems at a total Landau level filling factor $\nu=2$. The intersubband SDW dispersion is evaluated in the time-dependent Hartree-Fock approximation [5]. We find that the intersubband SDW modes could soften under experimentally accessible conditions, leading to a phase transition to a novel QHE state with interlayer inplane antiferromagnetic spin correlations $\left\langle S_{L}^{x}\right\rangle=-\left\langle S_{R}^{x}\right\rangle \neq 0(S$ is the electron spin operator, $\hat{x}$ is a direction parallel to the $2 \mathrm{D}$ plane with the magnetic field along the $\hat{z}$ direction, and $L$ and $R$ denote the left and right layers, respectively). Using a mean-field approximation, we are able to show that this antiferromagnetic phase is energetically stable and that the phase transition is continuous. We are, therefore, predicting a new quantum phase transition to a novel canted antiferromagnetic state in the double-layer system which occurs at zero temperature as system parameters (such as interlayer separation) are varied. 
Our findings seem to be consistent with recent inelastic light scattering measurements, [6] where a remarkable softening of the long wavelength SDW mode in a $\nu=2$ double quantum well system has been observed.

There has been a lot of work on double-layer QHE systems. Most studies [2,:7], however, have focused on $\nu=1$ (with some work [3] on $\nu=1 / 2$ ), leaving the $\nu=2$ state essentially uninvestigated. Our work shows that the $\nu=2$ QHE state, where the spin and the layer index compete with each other, has non-trivial magnetic properties. Although $\nu=1$ and $\nu=$ 2 double-layer QHE states exhibit some similarities such as the softening of the low energy collective excitations under certain conditions, there are important differences between them. At $\nu=1$, the spin degree of freedom is normally frozen out because of the $\mathrm{SU}(2)$ symmetry of the Coulomb interaction. The relevant low energy excitations in the $\nu=1$ QHE state are therefore intersubband charge-density-wave excitations, and the properties of the system are determined by the interplay between the interlayer tunneling energy and the Coulomb interaction energy. At $\nu=2$, both the spin degree of freedom and the layer degree of freedom are relevant, and the low energy excitations are intersubband SDW excitations. Consequently, the properties of the system are determined by the interplay among the tunneling energy, the Zeeman energy, and the Coulomb interaction energy. At $\nu=1$, the mode softening destroys the QHE [7] because beyond the critical layer separation the system is effectively a pair of isolated layers with compressible half-filled Landau level states, while at $\nu=2$, the QHE prevails in all phases due to the existence of incompressible filled Landau level states with charge excitation gaps, even at $d \rightarrow \infty$. The spin mode softening and the associated quantum phase transition to the canted antiferromagnetic state at $\nu=2$ are, however, experimentally observable through inelastic light scattering experiments [6].

In this work, we employ two different approaches to study the spin excitation instabilities in double-layer systems at $\nu=2$. These two approaches provide complementary information: one approach [5] deals with the collective excitations while the other [8] deals with the ground state properties. Both approaches are based on the Hartree-Fock approximation. In single-layer integer QHE systems, calculations [5] in the Hartree-Fock approximation agree 
well with experiments [9]. In double-layer systems, the Hartree-Fock approximation is less accurate because the Coulomb interaction potentials are more complicated. Nevertheless, we expect the Hartree-Fock approximation to remain reasonably good for a double-layer system at $\nu=2$, since the Hartree-Fock ground state, which is non-degenerate and separated in energy from higher levels, is a good approximation for the real many-body ground state at $\nu=2$ due to the existence of filled Landau levels and charge excitation gaps. Because our calculations employ realistic Coulomb interaction potentials (including finite well-thickness corrections [10]) and incorporate interlayer tunneling and Zeeman splitting, we expect our results to be not only qualitatively correct but also quantitatively reliable.

The Hamiltonian of the system is $\mathcal{H}=\mathcal{H}_{0}+\mathcal{H}_{\mathrm{I}}$ with

$$
\mathcal{H}_{0}=-\frac{\Delta_{\mathrm{sas}}}{2} \sum_{\alpha \sigma}\left(C_{1 \alpha \sigma}^{\dagger} C_{2 \alpha \sigma}+\text { h.c. }\right)-\frac{H}{2} \sum_{i \alpha \sigma} \sigma C_{i \alpha \sigma}^{\dagger} C_{i \alpha \sigma},
$$

where $C_{i \alpha \sigma}$ annihilates an electron in the lowest Landau level in layer $i(i=1,2)$ with spin $\sigma(\sigma= \pm 1)$ in the direction of the perpendicular field and with intraLandau level index $\alpha$. Interlayer tunneling induces the symmetric-antisymmetric energy separation $\Delta_{\text {sas }}$. The Coulomb interaction part of $\mathcal{H}$ is

$$
\begin{aligned}
\mathcal{H}_{\mathrm{I}}= & \frac{1}{2} \sum_{\sigma_{1} \sigma_{2}} \sum_{i j} \sum_{\alpha_{1} \alpha_{2}} \frac{1}{\Omega} \sum_{\mathbf{q}} V_{i j}(q) e^{-q^{2} l_{o}^{2} / 2} e^{i q_{x}\left(\alpha_{1}-\alpha_{2}\right) l_{o}^{2}} \\
& \times C_{i \alpha_{1}+q_{y} \sigma_{1}}^{\dagger} C_{j \alpha_{2} \sigma_{2}}^{\dagger} C_{j \alpha_{2}+q_{y} \sigma_{2}} C_{i \alpha_{1} \sigma_{1}},
\end{aligned}
$$

where $\Omega$ is the area of the sample. The interaction potentials are $V_{i j}=2 \pi e^{2} / \epsilon q F_{a}(q)$ for $i=j$ and $V_{i j}=\left(2 \pi e^{2} / \epsilon q\right) e^{-q d} F_{e}(q)$ for $i \neq j$. The finite-layer-thickness form factors $F_{a(e)}$ used in our calculations are taken from ref. [8].

We use $|\alpha \mu \sigma\rangle$ to denote the eigenstates of $\mathcal{H}_{0}$, where $\mu=0,1$ labels the symmetric and antisymmetric subbands. There are two intersubband SDW excitations which correspond to transitions $|0 \uparrow\rangle \leftrightarrow|1 \downarrow\rangle$ and $|0 \downarrow\rangle \leftrightarrow|1 \uparrow\rangle$. In the absence of interaction, these modes have excitation energies $\left|\Delta_{\text {sas }} \pm \Delta_{z}\right|$. The interaction renormalizes the excitation energies in two ways. One is due to the loss of exchange energy when an electron is excited to a higher but empty level, which raises the excitation energies. The other is an excitonic attraction 
between the electron excited to the higher level and the hole it leaves behind, which lowers the excitation energies. In diagrammatic perturbation theories, the effect of the exchange energy on the excitation energies is accounted for by including the corresponding self-energy in the electron Green's functions, and the effect of the excitonic attraction is represented by vertex corrections. The direct Hartree term does not influence the SDW excitations because the Coulomb interaction is spin-rotationally invariant. Since the Coulomb interaction potentials are subband-index dependent, they introduce mode-coupling between the two branches of the intersubband SDW excitations. This mode-coupling pushes down the frequency of the low-lying excitation and helps mode softening. The intersubband SDW excitation spectra are obtained as the poles of the retarded spin-density response function [5]

$$
\chi(q, \omega)=-i \int_{0}^{\infty} e^{i \omega t}\left\langle\left[\rho_{S D}(\mathbf{q}, t), \rho_{S D}^{\dagger}(-\mathbf{q}, 0)\right]\right\rangle
$$

where the intersubband SDW operator $\rho_{S D}$ is defined as follows. In the ferromagnetic ground state, i.e. when $|0 \uparrow\rangle$ and $|1 \uparrow\rangle$ are occupied, $\rho_{S D}(\mathbf{r})=\sum_{\mu} \psi_{\mu \uparrow}^{\dagger}(\mathbf{r}) \psi_{1-\mu \downarrow}(\mathbf{r})$, where $\psi_{\mu \sigma}$ annihilates an electron in subband $\mu$ with spin $\sigma$. In the symmetric ground state, i.e. when $|0 \uparrow\rangle$ and $|0 \downarrow\rangle$ are occupied, $\rho_{S D}(\mathbf{r})=\sum_{\sigma} \psi_{0 \sigma}^{\dagger}(\mathbf{r}) \psi_{1-\sigma}(\mathbf{r})$. Eq. (3) is evaluated in the time-dependent Hartree-Fock approximation [0], which we adapt to double-layer systems and, for simplicity, ignore all the higher Landau levels. As argued above, this should be a good approximation for our problem.

In Fig. 1, we show the dispersion of the intersubband SDW in the ferromagnetic ground state. As mentioned earlier, there are two intersubband SDW modes $\omega_{ \pm}(q)$ corresponding respectively to transitions $|0 \uparrow\rangle \rightarrow|1 \downarrow\rangle$ and $|1 \uparrow\rangle \rightarrow|0 \downarrow\rangle$. The frequencies $\omega_{ \pm}$increase as functions of $q$, approaching asymptotic values $\omega_{ \pm}(q \rightarrow \infty)=\omega_{ \pm}^{0}+\left|v_{x}\right|$, where $\omega_{ \pm}^{0}$ are the non-interacting excitation energies and $v_{x}$ is the exchange energy of an electron in the ground state. Mode coupling, which pushes down $\omega_{-}(q)$ and hence helps mode softening, is most visible at $q \rightarrow 0$. At zero layer separation, mode-coupling disappears, and we recover previously known results [5, (7]. The dispersions in Fig. 1 are shown for two different input parameters. In one case, there is no mode softening $\left(\omega_{ \pm}(q)>0\right)$, and in the other, there 
is mode softening $\left(\omega_{-}(q \rightarrow 0)<0\right)$. The mode softening signals that the ground state is unstable against spontaneous generation of intersubband SDW excitations. In Fig. 2, we show the intersubband SDW dispersion in the symmetric ground state. The results are qualitatively similar to those in Fig. 1. The important thing to notice is that there is mode softening here as well. We emphasize that our calculated SDW dispersion is experimentally measurable through depolarized inelastic light scattering experiments. In fact, the softening of the intersubband SDW excitation in a double-layer system at $\nu=2($ but NOT at $\nu=4)$ may have already been observed in a recent experiment [6].

The mode softening at $q \rightarrow 0$ suggests that the new phase has a broken symmetry: $\left\langle\rho_{S D}(q=0)\right\rangle \neq 0$, where $\rho_{S D}(q)$ is the intersubband SDW operator defined earlier. Since $\left\langle\rho_{S D}(q=0)\right\rangle=N_{\phi}\left[\left\langle S_{L}^{x}\right\rangle-\left\langle S_{R}^{x}\right\rangle\right]$, where $N_{\phi}$ is the Landau level degeneracy, the new phase is in fact characterized by an interlayer canted antiferromagnetic spin correlation. The phase diagram can be obtained by tracing the points where the mode softening occurs. The results are shown in Fig. 3. There are three phases in a double-layer QHE system at $\nu=2$ : a ferromagnetic phase where $|0 \uparrow\rangle$ and $|1 \uparrow\rangle$ are occupied, a symmetric phase where $|0 \uparrow\rangle$ and $|0 \downarrow\rangle$ are occupied (it may also be viewed as ferromagnetic in the sub-space associated with the layer degrees of freedom [2]), and the antiferromagnetic phase. The symmetric phase exists for $\Delta_{\text {sas }}>\Delta_{z}$ and $d<d_{c 1}$, the antiferromagnetic phase exists for $\Delta_{\text {sas }}>\Delta_{z}$ and $d_{c 1}<d<d_{c 2}$, and the ferromagnetic phase exists for either $\Delta_{z}>\Delta_{\text {sas }}$ or $d>d_{c 2}$. The ferromagnetic phase is favored when $\Delta_{z}$ is increased, while the symmetric phase is favored when $\Delta_{\text {sas }}$ is increased. It should be noted that, for a given $\Delta_{s a s}, d_{c 1}$ is considerably smaller than the critical layer separation where the charge density excitation in the $\nu=1$ state becomes soft [7]. The reason for this is the absence of the Hartree contribution to SDW excitations. An intuitive explanation on the existence of the canted antiferromagnetic phase is that in this phase the system can take advantage of the tunneling energy ( the kinetic energy in the perpendicular direction) by having an inter-layer antiferromagnetic spin correlation - an example of the super-exchange induced antiferromagnetic correlation. The reason the antiferromagnetic ordering is canted is to minimize Zeeman energy. We 
emphasize that our predicted phase diagram should be experimentally measurable.

The antiferromagnetic ground state can also be calculated within a mean-field approximation. The approach we employ is the same as that used to study magnetic-field-induced Wigner solid phase in 2D systems [8], except that we only look for uniform solutions and allow for the possibility of a non-zero antiferromagnetic order parameter $\left(\left\langle\rho_{S D}(q=0)\right\rangle \neq 0\right)$. The energies obtained from this mean-field theory show that the antiferromagnetic phase is energetically stable. In Fig. 4, we show the antiferromagnetic order parameter obtained in this mean-field approximation. Several features are obvious from this figure: (i) The antiferromagnetic phase exists only at intermediate layer separations. (ii) The range of layer separations in which the antiferromagnetic phase exists shrinks with increasing Zeeman energy. (iii) The phase transitions are continuous. This mean-field theory also provides a way to derive the phase diagram, and the phase diagram obtained in this manner is identical to the one obtained from the softening of the intersubband SDW excitations, providing a confirmation of our predictions.

Our calculations at $\nu=2$ are well controlled in the absence of Landau level mixing. We expect that the Landau level mixing is negligible at $\nu=2$, but may not be negligible at $\nu=6$ (otherwise, the results presented here would be qualitatively valid at $\nu=6[11]$ ). We would like to emphasize that the Hartree-Fock approximation fails completely at non-integer filling factors, because the ground states are macroscopically degenerate. Nevertheless, some qualitative speculations for non-integer filling factors can be made. For example, when $\nu$ is changed away from 2, the presence of screening, which reduces the electron-hole excitonic attraction, may increase the intersubband SDW energy and prevent the mode softening. Thus, the antiferromagnetic phase would be unstable away from $\nu=2$. This is precisely the experimental observation in ref. [6] where a sharp minimum in the SDW energy is found at $\nu=2$.

In summary, we have studied the instabilities induced by the softening of intersubband spin-density-wave excitations in double-layer quantum Hall systems at $\nu=2$ within the timedependent Hartree-Fock approximation. The intersubband spin-density excitation modes 
soften under experimentally accessible conditions and lead to a novel quantum Hall state with interlayer planar antiferromagnetic spin correlations. We show, in a mean-field approximation, that this planar antiferromagnetic phase is energetically stable and that the phase transition is continuous. We, therefore, predict the existence of a novel canted antiferromagnetic phase under suitable conditions in between the symmetric and the ferromagnetic phases in a double-layer QHE sample at $\nu=2$.

The authors thank Dr. A. Pinczuk for helpful discussions on the experimental data. This work is supported by the U.S.-ARO and the U.S.-ONR. 


\section{REFERENCES}

[1] Quantum Hall Effect, edited by R.E. Prange and S.M. Girvin (Springer-Verlag, New York, 1987); Quantum Hall Effect: A Perspective, edited by A.H. MacDonald (Kluwer, Dordrecht, 1990); Perspectives in Quantum Hall Effects, edited by S. Das Sarma and A. Pinczuk (Wiley, New York, 1996).

[2] S.Q. Murphy et al., Phys. Rev. Lett. 72, 728 (1994); K. Yang et al., ibid. 72, 732 (1994); K. Moon et al., Phys. Rev. B. 51, 5138 (1995), and references therein.

[3] Y.W. Suen et al., Phys. Rev. Lett. 68, 1379 (1992); J.P. Eisenstein et al., ibid. 68, 1383 (1992); D. Yoshioka, A.H. MacDonald, and S.M. Girvin, Phys. Rev. B. 39, 1932 (1989); Song He, S. Das Sarma, and X.C. Xie, ibid. 47, 4394 (1993).

[4] S. Das Sarma and P.I. Tamborenea, Phys. Rev. Lett. 73, 1971 (1994); R.J. Radtke, P.I. Tamborenea, and S. Das Sarma, Phys. Rev. B 54, 13832 (1996).

[5] C. Kallin and B.I. Halperin, Phys. Rev. B. 30, 5655 (1984).

[6] V. Pellegrini et al., Phys. Rev. Lett. 78, 310 (1997).

[7] A.H. MacDonald, P.M. Platzman, and G.S. Boebinger, Phys. Rev. Lett. 65, 775 (1990); L. Brey, ibid. 65, 903 (1990); H.A. Fertig, Phys. Rev. B. 40, 1087 (1989); X.M. Chen and J.J. Quinn, ibid. 45, 11054 (1992).

[8] R. Côté, L. Brey, and A.H. MacDonald, Phys. Rev. B. 46, 10239 (1992); L. Zheng and H.A. Fertig, ibid. 52, 12282 (1995).

[9] A. Pinczuk et al., Phys. Rev. Lett. 68, 3623 (1992).

[10] The finite well-thickness corrections, as well as other detailed corrections to interaction potentials, have quantitative effects only. They do not change any results qualitatively.

[11] On the other hand, the situation at $\nu=4$ is very different from the situation at $\nu=2$, since the inter-Landau level excitation energies are comparable to the cyclotron energy. 


\section{FIGURES}

FIG. 1. Inter-subband SDW dispersion $\omega_{ \pm}(q)$ in the ferromagnetic (FM) phase at $\nu=2$. The solid lines are for $\Delta_{\text {sas }}=0.02 e^{2} / \epsilon l_{o}$, and the dashed lines are for $\Delta_{\text {sas }}=0.1 e^{2} / \epsilon l_{o}$. Other parameters are the same for both the solid and dashed lines: the Zeeman energy $\Delta_{z}=0.01 e^{2} / \epsilon l_{o}$, the layer separation $d=1.15 l_{o}$, and the well-thickness $d_{w}=0.8 l_{o}$. Notice that $\omega_{-}(q \rightarrow 0)<0$ for $\Delta_{\text {sas }}=0.1 e^{2} / \epsilon l_{o}$ (dashed line).

FIG. 2. Inter-subband SDW dispersion $\omega_{ \pm}(q)$ in the symmetric (SYM) phase at $\nu=2$. The solid lines are for layer separation $d=0.85 l_{o}$ and the dashed lines are for $d=0.95 l_{o}$. Other param-

eters are the same for both the solid lines and dashed lines: $\Delta_{z}=0.08 e^{2} / \epsilon l_{o}, \Delta_{\text {sas }}=0.35 e^{2} / \epsilon l_{o}$, $d_{w}=0.8 l_{o}$. Notice that $\omega_{-}(q \rightarrow 0)<0$ for $d=0.95 l_{o}$ (dashed line).

FIG. 3. Phase diagrams at $\nu=2$ for two different Zeeman energies: (a) $\Delta_{z}=0.01 e^{2} / \epsilon l_{o}$ and (b) $\Delta_{z}=0.08 e^{2} / \epsilon l_{o}$. The well-thickness $d_{w}=0.8 l_{o}$ in both cases. Three phases are present: a symmetric phase (SYM), a ferromagnetic phase (FM), and an antiferromagnetic phase (AF). The ' $\times$ ' in (a) denotes the experimental sample parameters of Ref. 6 (with a magnetic field $B=1.3 T$ ), where the measured SDW energy has a sharp minimum at $\nu=2$ with a value of 0.04 meV which is comparable to the experimental temperature of $0.6 \mathrm{~K}$.

FIG. 4. Antiferromagnetic order parameter $\left\langle\rho_{S D}(q=0)\right\rangle / N_{\phi}$ versus layer separation $d$ for the indicated Zeeman energies, where $N_{\phi}$ is the Landau level degeneracy and $\rho_{S D}(q)$ is the intersubband spin-density operator defined in the text. The well-thickness $d=0.8 l_{o}$. 
Fig1 Zheng, Radtke, Das Sarma

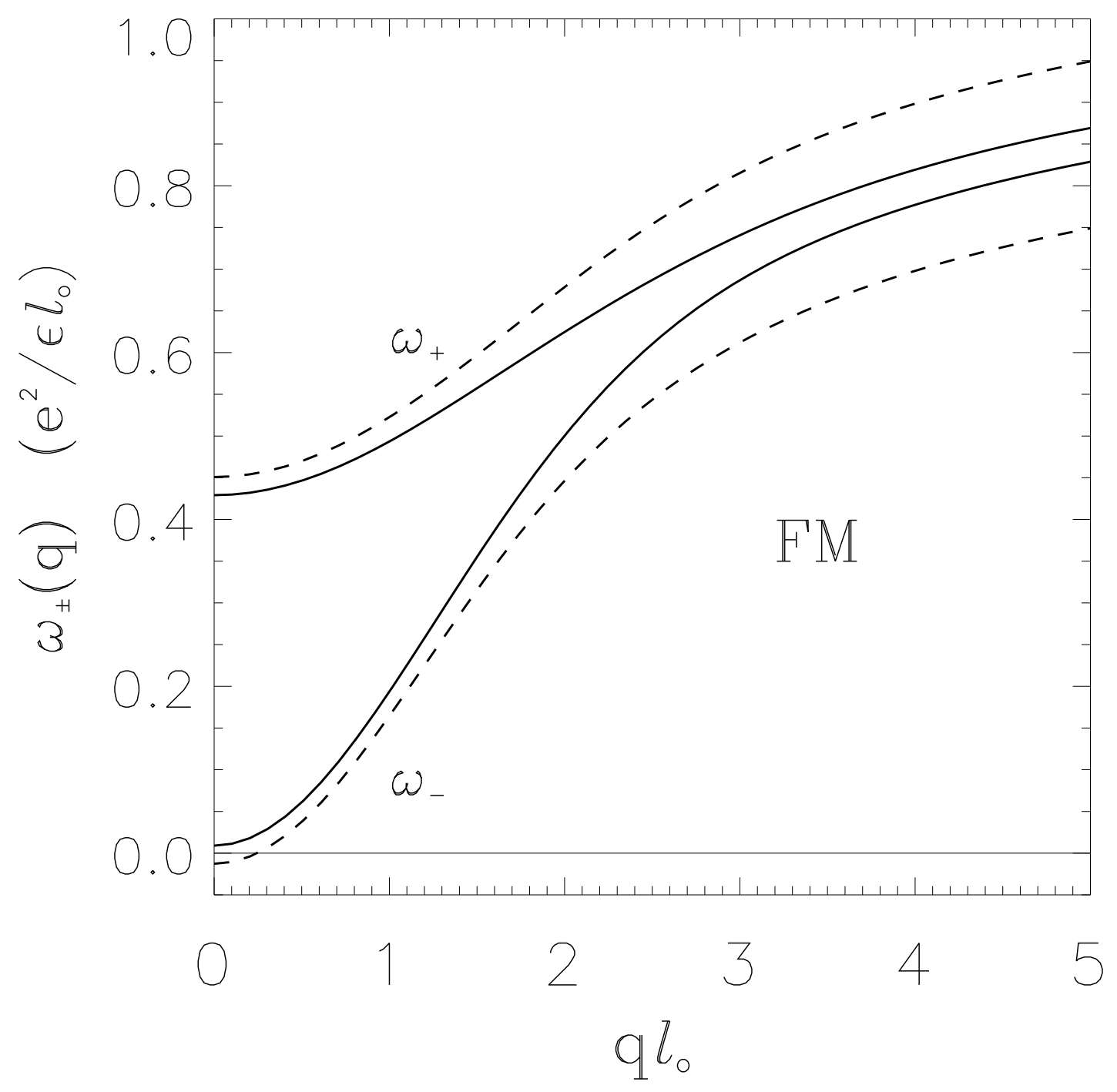


Fig2 Zheng, Radtke, Das Sarma

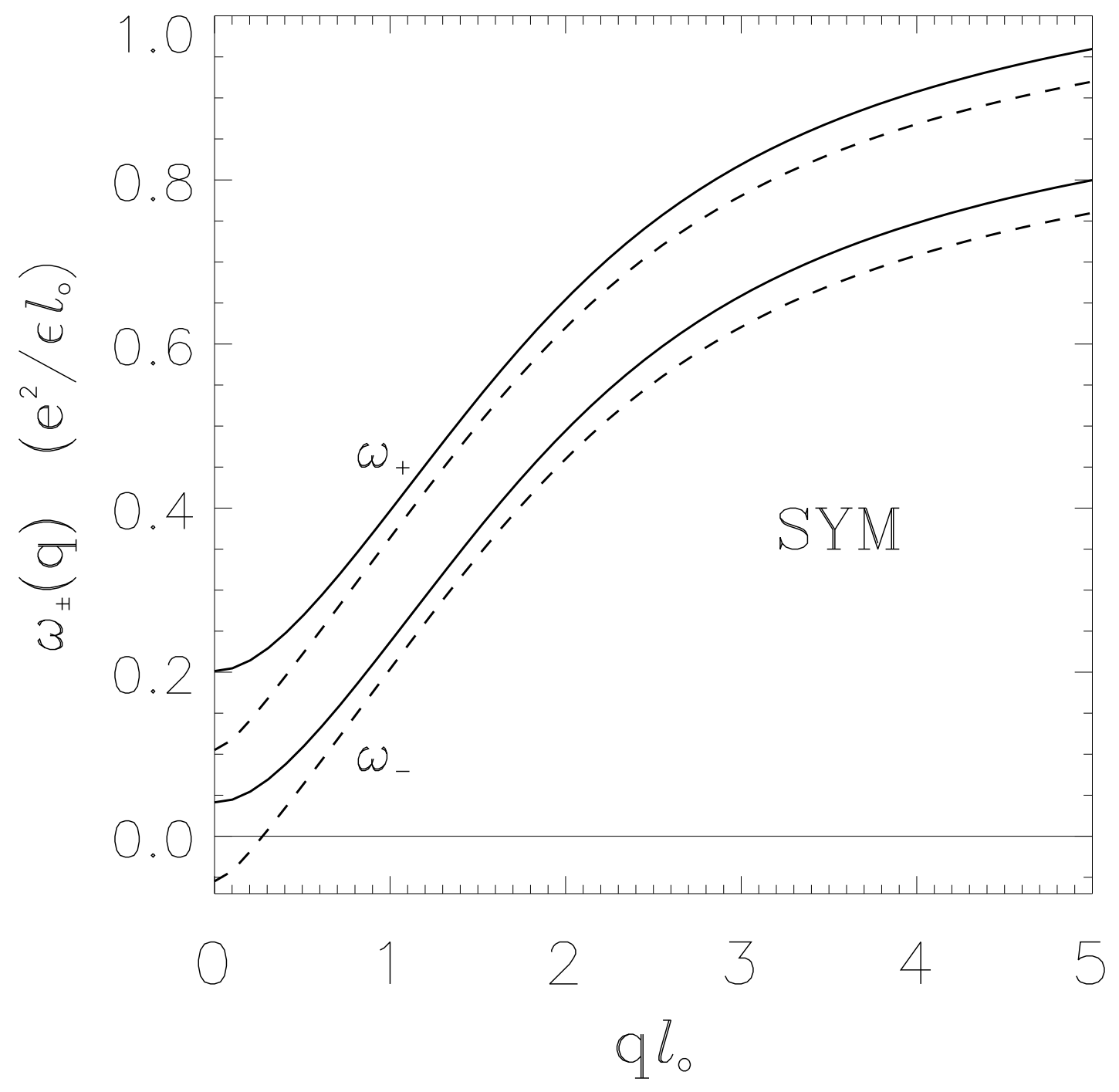


Fig3a Zheng, Radtke, Das Sarma

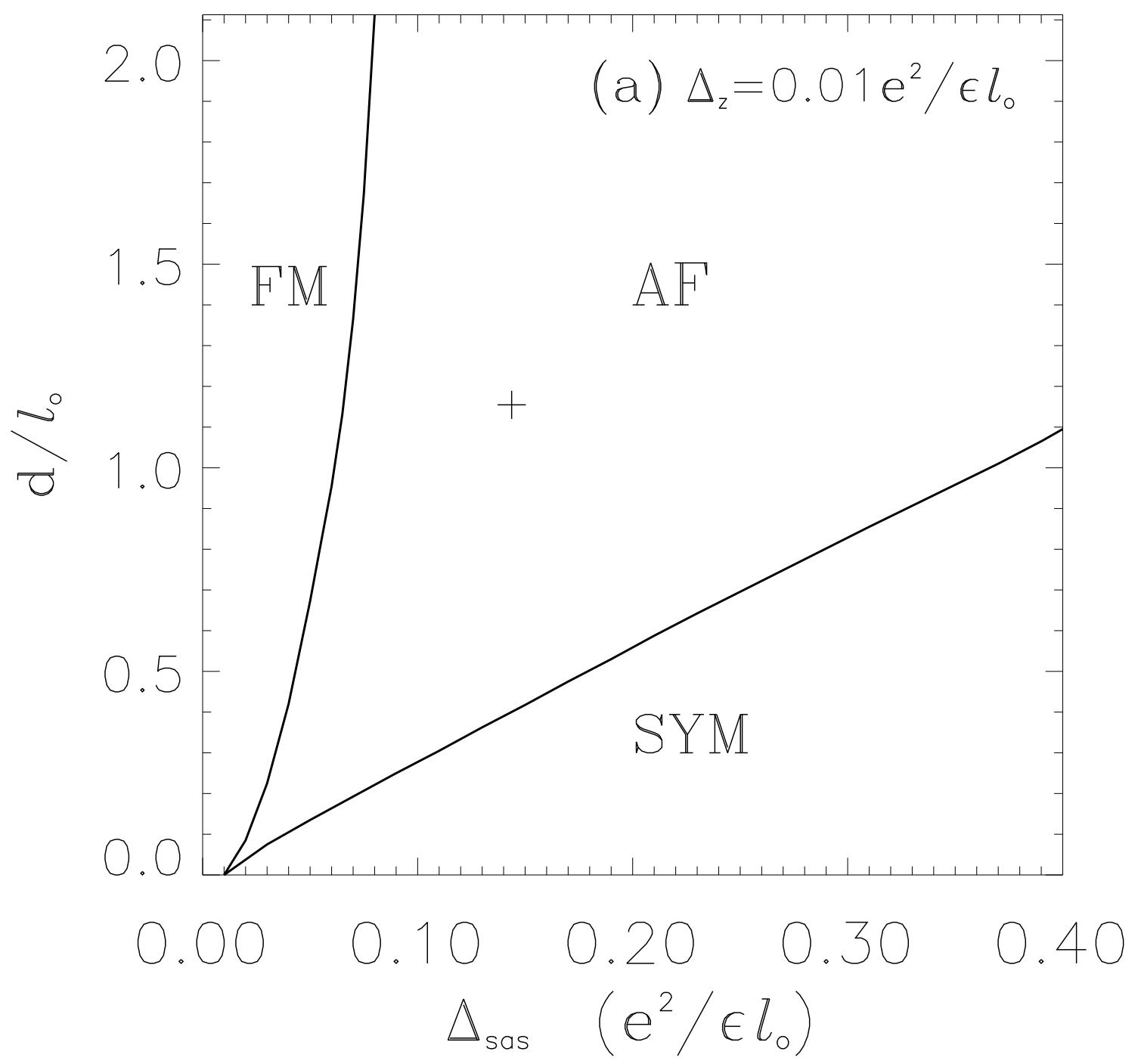


Fig3b Zheng, Radtke, Das Sarma

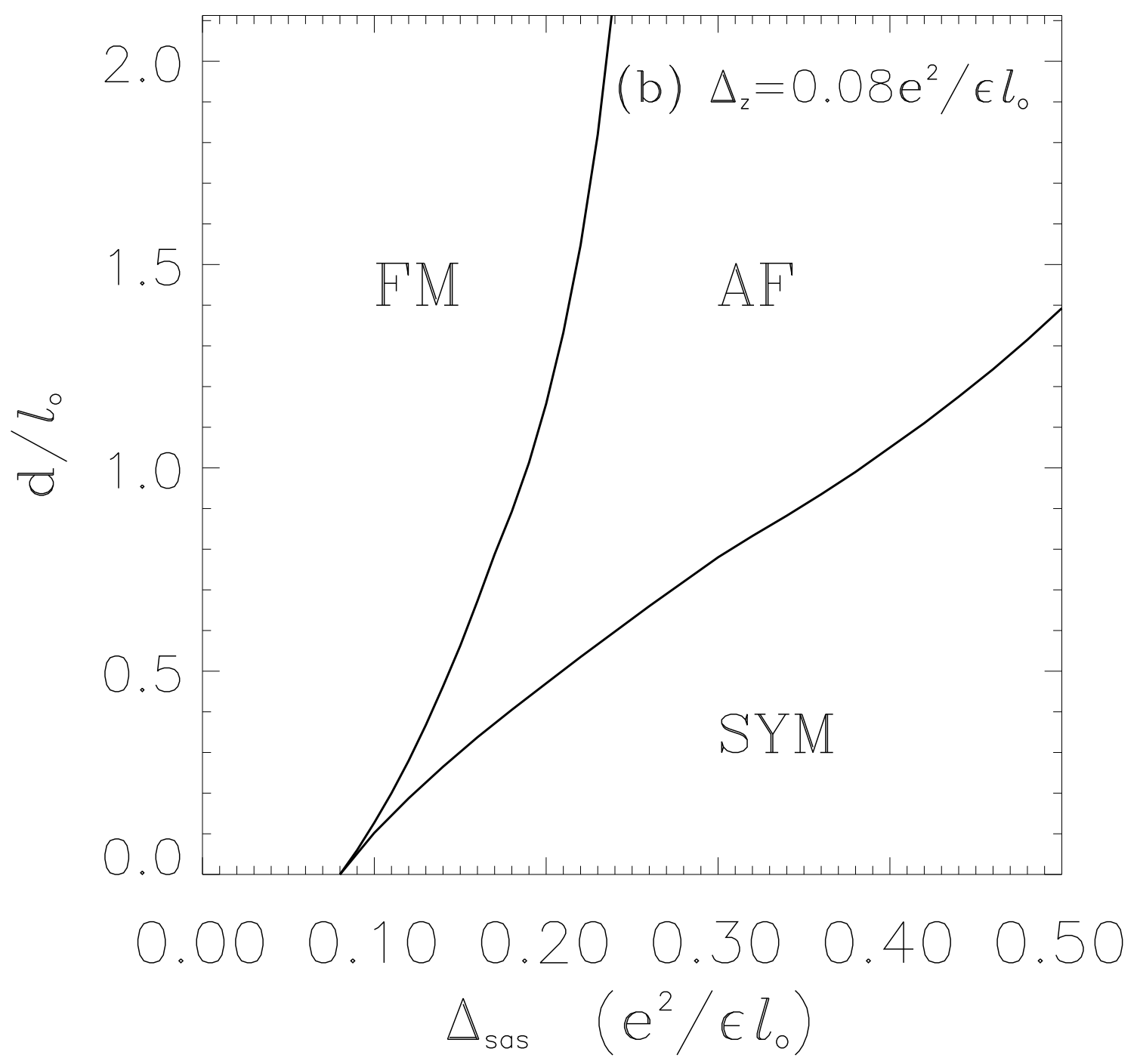


Fig4 Zheng, Radtke, Das Sarma

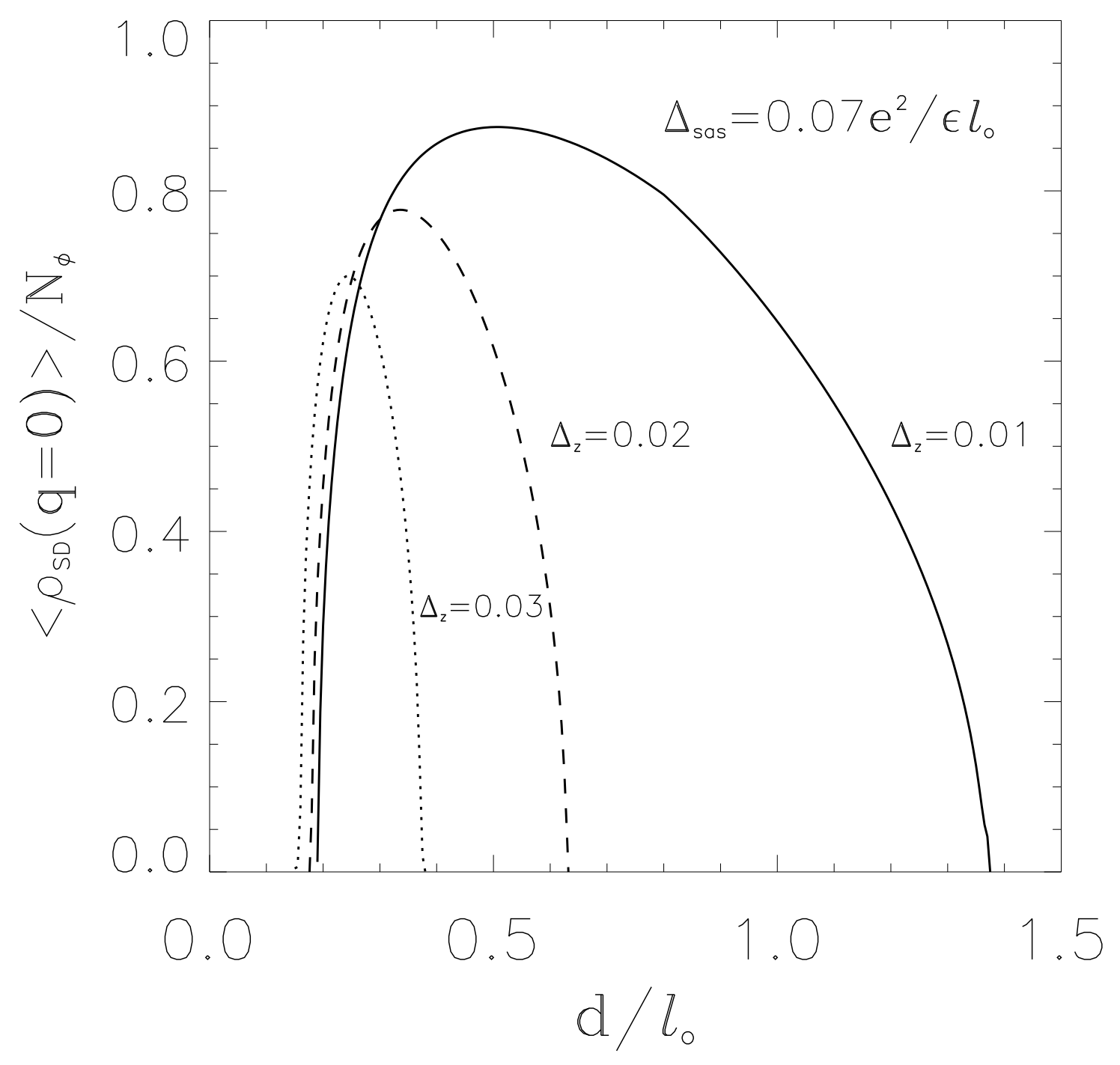

